\title{
Twin pregnancy with complete hydatidiform mole and coexisting live fetus complicated with HELLP syndrome
}

\author{
Deepti Sharma*, M. G. Usha, Ramesh Gaikwad
}

Department of Obstetrics \& Gynecology, Amrita Institute of Medical Sciences, Kochi-682041, Kerala, India

Received: 15 December 2012

Accepted: 6 January 2013

*Correspondence:

Dr. Deepti Sharma

E-mail: sdeepti108@gmail.com

\begin{abstract}
Hydatidiform mole with a coexisting live twin is an extremely rare event. It poses a great challenge for the obstetrician to decide for or against continuation of such a pregnancy, especially if the conception is after infertility treatment. Continuation carries risk of medical complications and persistent trophoblastic disease. We report a case of 22 year old primigravida, with complete hydatidiform mole coexisting with a live fetus. Patient had conceived in her fourth cycle of ovulation induction and wanted to continue pregnancy. She developed severe preeclampsia and HELLP syndrome. In this case report we highlight the importance of intense antenatal monitoring and the possibility of sudden deterioration of maternal condition forcing immediate termination.
\end{abstract}

Keywords: Hydatidiform mole, Coexisting fetus, Dizygotic twins

\section{INTRODUCTION}

Molar pregnancy with a coexisting live twin is an extremely rare entity. The incidence reported in literature varies from 1 in 10,000 to 1 in 100,000 pregnancies. ${ }^{1}$ Although successful outcomes in the form of live births have been reported, continuation of pregnancy till term is usually uncommon. Management of these cases is a clinical dilemma as it carries the risk of severe maternal complications like preeclampsia, thyrotoxicosis, antepartum haemorrhage and persistent trophoblastic disease. Fetus also entails the risks of malformations, chromosomal abnormalities and extreme prematurity. The choice of termination of pregnancy as against continuation is extremely difficult, particularly if the conception follows a long period of infertility and results after assisted reproduction.

\section{CASE REPORT}

A 22 year old primigravida was referred from local hospital at 14 weeks gestation, with the diagnosis of complete hydatidiform mole with coexisting viable twin for further management. She had been married for 2-1/2 years and had undergone evaluation for primary infertility. She underwent 3 cycles of ovulation induction with $50 \mathrm{mg}$ clomiphene citrate previously. She conceived in her fourth cycle of induction with clomiphene combined with gonadotropins. The index pregnancy was closely monitored with serial ultrasound examination. The initial scan at 4 weeks revealed 3-4 mm twin GS, at 9 weeks showed a live $8 \mathrm{wk}^{+5}$ gestation coexisting with an empty $20 \mathrm{~mm}$ twin sac (reported as dizygotic twins). Last scan at 14 weeks showed a $13 \mathrm{wk}^{+5}$ day live fetus with the empty twin sac containing a mixed echogenic mass measuring $11 \mathrm{X} 6 \mathrm{~cm}$, with multiple small cysts of varying sizes (Figure 1).

After detailed counseling regarding the maternal and fetal risks, patient opted for continuation of pregnancy. Her antenatal history till date was significant for recurrent episodes of hyperemesis gravidarum and no history of vaginal bleeding. On admission, her vitals were stable and had an anxious look due to repeated episodes of vomiting. Uterus was enlarged to 16 weeks size with normal fetal heart tones. Investigations showed a normal hemogram, liver function and renal function tests. Beta HCG on admission was 2,51,500 $\mathrm{mIU} / \mathrm{ml}$. Thyroid function tests were deranged and she was started on propylthiouracil $100 \mathrm{mg}$ tid in view of hyperthyroidism. She was managed conservatively for hyperemesis 
gravidarum with intravenous fluids and electrolyte correction.

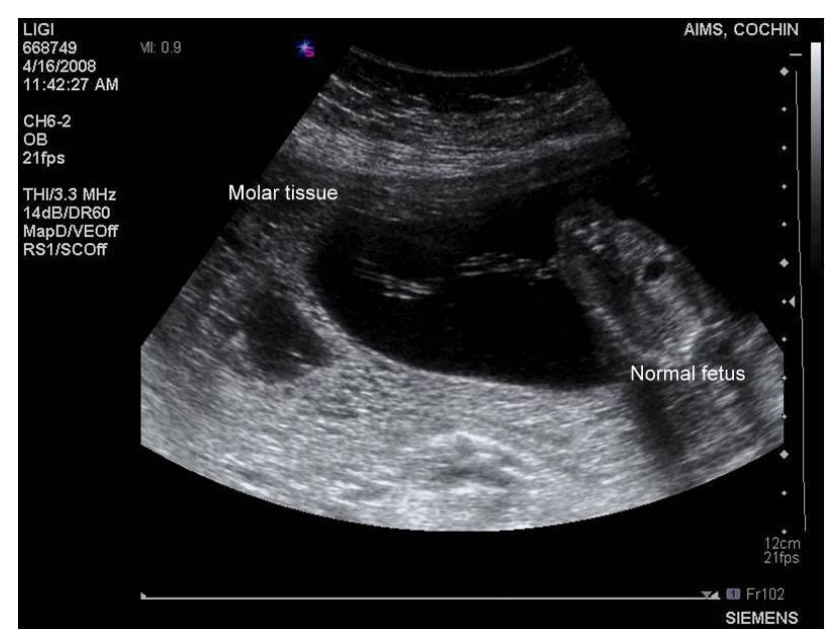

Figure 1: Molar tissue demonstrating classical snow storm appearance seen to the right of a normal live fetus.

USG guided aspiration biopsy was done from the molar tissue through the transabdominal route. It showed hydropic villi with no evidence of atypia. Genetic amniocentesis and karyotyping of the live fetus was planned at 16 weeks of gestation. Within a week she showed an increase of uterine size to 18 weeks and developed slight vaginal discharge with brownish black discoloration, but no active vaginal bleeding. Intense antenatal monitoring was done with careful watch on clinical signs, blood pressure and proteinuria, serum electrolytes, TFT and liver function tests.

At $15 \mathrm{wk}^{+5}$ she developed hypertension without proteinuria and was managed with anti-hypertensives. Within $48 \mathrm{hrs}$ of onset of hypertension her lab parameters showed deranged liver function tests and severe drop in platelet counts. Immediate termination was undertaken in view of early onset preeclampsia progressing rapidly to HELLP syndrome. Patient was euthyroid and serum electrolytes were normal prior to evacuation. Termination was done under GA using suction evacuation and specimen was sent for histopathological examination. She received one unit of packed red cells in the postoperative period.

Post-evacuation serial beta HCG monitoring was done at weekly interval and showed a normal HCG regression curve. The levels became negative within six weeks. She underwent a complete follow up of hydatidiform mole for one year which was unremarkable.

\section{DISCUSSION}

Hydatidiform mole with a coexisting live twin is an extremely rare event. It poses a great challenge for the obstetrician to decide for or against continuation of such a pregnancy, especially if the conception is after infertility treatment. The condition can result due to two mechanisms; a complete mole along with a normal diploid fetus, and a partial mole with a triploid fetus. The differentiation of the two types is important as the perinatal outcomes of the two are different. The abnormal triploid fetus coexisting with a partial mole tends to die in the first trimester, while the diploid fetus with a complete mole in the dizygotic twin pregnancy has a fair chance to survive. ${ }^{2}$ Thus, the demonstration of a normal karyotype of the fetus is essential, especially if the diagnosis is discovered late in the gestation.

The natural history of the condition is unclear and optimal management guidelines are not clearly outlined. ${ }^{3}$ The number of reported cases is also on the rise due to widespread use of ovulation induction, advances in ultrasonography and availability of cytogenetic studies. In the past such cases were invariably terminated soon after the diagnosis due to the risk of malignant transformation and serious maternal medical complications. It has been reported that the chance of continuation of the pregnancy beyond 28 weeks of gestation is around $60 \%$. $^{4}$ Of these pregnancies, fetal survival is reported in about $70 \%$ cases and $30 \%$ chance of fetal loss due to intrauterine or early neonatal death. $15 \%$ of the cases may require sudden interruption of the pregnancy due to severe complications like preeclampsia or APH. The incidence of PTD is reported to be $19 \%$ in patients who decided for termination in contrast to $9 \%$ in those who continued. ${ }^{4}$ Advanced gestational age is not linked to increase in persistent trophoblastic disease. No reports have demonstrated an increase in the risk of invasive mole or choriocarcinoma with progressing gestational age. ${ }^{5}$ Maternal serum HCG could be used as a marker in predicting the outcome of such cases and its role has to be further evaluated through prospective studies. ${ }^{6}$

It is prudent to allow the couple to make an informed choice. Couple should be thoroughly counseled regarding the maternal and fetal risks. It should be particularly stressed that the continuation involves intensive ante- and post-partum surveillance. The likelihood of persistent trophoblastic disease along with the possible requirement of chemotherapy should also be explained. The pregnancy should be closely monitored by a perinatal team with experience in high risk obstetrics and a good intensive neonatal care.

\section{CONCLUSION}

Every pregnancy outcome is not a success story. We attempted continuation of this extremely desired pregnancy with the knowledge of all possible complications. The molar pregnancy turned out to be "an evil twin" as its associated complications compelled to change the decision towards termination before the healthy fetus could reach the age of viability. 
Funding: No funding sources

Competing interests: There are no competing interests to declare

Ethical approval: Not required

\section{REFERENCES}

1. Cunningham ME, Walls WL, Burke MF. Gray scale ultrasonography in the diagnosis of hydatidiform mole with a coexisting fetus. Br J Obstet Gynecol 1977;84:73-5.

2. Jauniaux E. Ultrasound diagnosis and follow up of gestational trophoblastic disease. Ultrasound Obstet Gynecol 1998;11:367-77.
3. Kafali H, Cengiz B. Hydatidifrom mole with coexisting twin after in-vitro fertilization. Internet $\mathbf{J}$ Gynecol Obstet 2003;2(2).

4. Vejerslev LO. Clinical management and diagnostic possibilities in hydatidiform mole with coexistent fetus. Obstet Gynecol Surv 1991;46:577-88.

5. Chen FP. Molar pregnancy and living normal fetus coexisting until term: prenatal biochemical and sonographic diagnosis. Hum Reprod 1997;12:853-6.

6. Wee L, Jauniaux E. Prenatal diagnosis and management of twin pregnancies complicated by a co-existing molar pregnancy. Prenat Diagn 2005;25:772-6.

DOI: $10.5455 / 2320-1770 . i j r \operatorname{cog} 20130217$

Cite this article as: Sharma D, Usha MG, Gaikwad

$\mathrm{R}$. Twin pregnancy with complete hydatidiform mole and coexisting live fetus complicated with HELLP syndrome. Int J Reprod Contracept Obstet Gynecol 2013;2:92-4. 